\author{
Omega-P, Inc. \\ 258 Bradley Street, Suite 200 \\ New Haven, CT 06510 \\ Final Report to US Department of Energy \\ SBIR Grant DE-FG02-06ER84472
}

\title{
FAST FERROELECTRIC L-BAND TUNER FOR ILC CAVITIES
}

\section{CONTENTS}

\begin{tabular}{|c|c|}
\hline I. & INTRODUCTION \\
\hline II. & TECHNICAL APPROACH \\
\hline III. & PLANAR GEOMETRY FERROELECTRIC TUNER CONCEPT \\
\hline & IIIa. Simulation of tuner performance \\
\hline & IIIb. Measurements of loss tangent of ferroelectric material \\
\hline & IIIc. Bench tests of one-third model of planar geometry ferroelectric tunes \\
\hline & IIId. Measurements of tuner loss \\
\hline & IIIe. Measurements of switching speed \\
\hline & IIIf. Alternative phase shifter concept: a radial line reflector \\
\hline & ERENCES \\
\hline
\end{tabular}

\begin{abstract}
Design, analysis, and low-power tests are described on a $1.3 \mathrm{GHz}$ ferroelectric tuner that could find application in the International Linear Collider or in Project X at Fermi National Accelerator Laboratory. The tuner configuration utilizes a three-deck sandwich imbedded in a WR-650 waveguide, in which ferroelectric bars are clamped between conducting plates that allow the tuning bias voltage to be applied. Use of a reduced one-third structure allowed tests of critical parameters of the configuration, including phase shift, loss, and switching speed. Issues that were revealed that require improvement include reducing loss tangent in the ferroelectric material, development of a reliable means of brazing ferroelectric elements to copper parts of the tuner, and simplification of the mechanical design of the configuration.
\end{abstract}

Work on this project was carried out by senior research scientist Dr. S.Yu. Kazakov, with important input from consultant Dr. V.P. Yakovlev of FNAL and research scientist Dr. S.V. Shchelkunov of Yale University, with Dr. J.L. Hirshfield serving as Principal Investigator. The report was written by Dr. Hirshfield.

March 12, 2010 


\section{Omega-P, Inc.}

Final report to DoE on Phase II SBIR grant DE-FG02-06 ER 84472

FAST FERROELECTRIC L-BAND TUNER FOR ILC CAVITIES

\section{INTRODUCTION}

Under Topic 37a in the DoE 2006 SBIR Program Solicitation, proposals were sought "...for technology to support the development of fundamental power couplers and tuners for 1.3 $\mathrm{GHz}$ superconducting RF cavities. Areas of interest include improvements to current coupler design (resulting in reduced conditioning time, reduced cost, and improved reliability), as well as new tuner designs and concepts." Omega-P, Inc. submitted Phase I and subsequently Phase II proposals entitled "Fast Ferroelectric L-Band Tuner For ILC Cavities," and was awarded Phase II grant DE-FG02-06 ER 84472. This report summarizes work accomplished during the project. The overall goal of the R\&D program was to design, engineer, build, and test an L-band externally-controlled fast ferroelectric tuner for controlling the coupling of superconducting RF cavities for a future linear collider ILC [1]. Phase II was carried out in a collaboration led by Omega-P that involves Euclid Techlabs LLC, which carries out development of the ferroelectric materials and supplied the ferroelectric elements for the tuner. Eventually, it is anticipated that the Technical Division of Fermi National Accelerator Laboratory will make facilities available for high-power tests of the tuner and will participate jointly in the tests. Such a tuner could be of major significance for ILC and Project X.

Successful demonstration of this tuner is anticipated in future under power and pulselength conditions that are specified in the February 7, 2007 ILC Reference Design Report (RDR) for the $1.3 \mathrm{GHz}$ superconducting cavities in ILC. This tuner will enable RF power control to be introduced at each cavity with the capability of fast tuning to compensate for Lorentz force cavity distortions and microphonics that can de-tune and de-phase the RF cavity fields. The tuner can also introduce programmed coupling variations during the RF pulse to reduce RF power demand, thereby providing reduction in the AC mains power demand. Development of the tuner proposed here is fully consistent with the RDR where it states in para 3.6.2.4: "Currently no tuner has been chosen for the baseline design, and R\&D is required to determine the reliability and installed performance of current designs."

As described in the RDR, the RF system for ILC includes 21,024 one-meter long 9-cell superconducting cavities. Groups of 12 such cavities would be installed in a common cryostat. The accelerating gradient is to be about $35 \mathrm{MeV} / \mathrm{m}$ and the c.m. energy is to be $800 \mathrm{GeV}$. The $\mathrm{RF}$ power is generated by 620 klystrons per linac, each feeding 36 nine-cell cavities. The required peak power per klystron is about $10 \mathrm{MW}$, including a $10 \%$ overhead for correcting phase errors during the beam pulse which arise from Lorentz force detuning and microphonics. The RF pulse length is $1.28 \mathrm{~ms}$, which includes a beam pulse length of $860 \mu \mathrm{s}$, and a cavity fill time of $420 \mu \mathrm{s}$. The repetition rate is $5 \mathrm{~Hz}$. The average AC mains power consumed by the RF system at $800 \mathrm{GeV}$ c.m. energy is about $150 \mathrm{MW}$, taking into account an RF source efficiency of $\sim 65 \%$ [3-5], and a modulator efficiency of $85 \%$ [6]. Refrigerators take an additional $27.6 \mathrm{MW}$, 


\section{Omega-P, Inc.}

Final report to DoE on Phase II SBIR grant DE-FG02-06 ER 84472

FAST FERROELECTRIC L-BAND TUNER FOR ILC CAVITIES

to dissipate heat from RF losses in the structures, plus static heat load compensation and heat from quadrupoles of $8.7 \mathrm{MW}$ [7].

Severe requirements for stability of RF phase during the pulse in ILC acceleration sections exist: the r.m.s. phase deviation should be no greater than $0.4^{\circ}$ [8]. One can identify a number of sources of uncontrolled phase changes in the cavities. First of all, the half-length $L$ of the power distribution system of one section fed by one klystron is quite long, about $20 \mathrm{~m}$. Reflections in components along the distribution line (directional couplers, circulators, etc) may cause significant phase spread in the cavities of each section, especially if breakdown occurs in one cavity. It can be noted that a waveguide temperature change of $1^{\circ} \mathrm{C}$ will cause a phase difference $\Delta \varphi$ of $0.6^{\circ}$ in a section of cavities $\left[\Delta \varphi \approx 720^{\circ} \times \exp (-5 \Delta L / \lambda)\right.$ where $\lambda$. is wavelength]. Another possible reason for phase deviation is a cavity resonance frequency variation due to small changes in the cavity geometry because of mechanical vibrations, or socalled microphonics. In addition, the resonant frequency changes during cavity filling because of Lorentz forces, and spread of so-called cavity Lorentz factors caused by difference in mechanical properties of the cavities may give additional uncontrolled phase errors.

There are two strategies that can be used in order to confront problems caused by microphonics and Lorentz forces [5]. The first is to apply an active correction to the cavity dimensions using a piezoelectric (or magnetostrictive) frequency tuner [6,7]. This piezoelectric tuner changes the cavity length such a way that the detuning caused by microphonics is nearlyperfectly compensated. Due to the partially statistical nature of microphonics noise, a negative feedback loop is necessary. The device must allow correction of micron-scale cavity deformations at frequencies up to about $100 \mathrm{~Hz}$. The piezoelectric tuner allows one to maintain a cavity's resonance frequency by applying a correction directly to the source of the perturbing influence, i.e., to counteract variations in the cavity length. However the piezoelectric tuner must be operated at cryogenic temperatures and thus permits only limited access inside the cryomodule in the event of a failure. Further, the piezoelectric device has its own mechanical resonances which may interfere with control system performance if the self-resonance frequency overlaps with the microphonic excitation to be controlled [5]. In addition, it may be a problem in this case to compensate uncontrolled external phase errors caused by perturbations in the long power distribution system. A second means for neutralizing microphonics and Lorentz force is to use an external tuner to apply a corrective phase shift to the reflected RF wave and reintroduce it to the cavity structure [8,9]. Early on, ferrite tuners were suggested for this application $[10,11,12]$. High-power fast ferrite tuners are currently being developed at CERN for the Superconducting Proton Linac [9] operating at $352 \mathrm{MHz}$. This CERN ferrite tuner is designed to provide fast phase and amplitude modulation of the drive signal for individual superconducting cavities. The ferrite tuner is based on two fast, compact high-power ferrite phase shifters magnetically biased by external coils. Another tuner was developed and successfully tested at Fermilab [12]. The tuning frequency for ferrite tuners will have an upper cut-off at $\sim 2 \mathrm{kHz}$ that 


\section{Omega-P, Inc.}

Final report to DoE on Phase II SBIR grant DE-FG02-06 ER 84472

FAST FERROELECTRIC L-BAND TUNER FOR ILC CAVITIES

comes mainly from eddy currents inside the RF structure [11]. It may be important that an external tuner allows compensation of the phase errors caused by the power distribution system. However, ILC has a stringent requirement for amplitude and phase stability. Thus, the gain in the control feedback loop must be high enough, and its bandwidth wide enough, to insure this high degree of stability. This may rule out ferrite tuners with their narrow bandwidth.

The RF power requirement for cavities in a SRF linac is determined by the accelerating gradient maintained in the cavity and the beam loading. Additional power is required for corrections of phase errors which arise from Lorentz force detuning, and other uncontrolled sources of detuning $[3,4]$. In ERLs the beam loading is very small, and the power requirements are determined by the Ohmic looses in the cavity walls, by unbalanced beam currents, and by the cavity resonance frequency variation due to small changes in the cavity geometry because of mechanical vibrations. Solution to the last problem, so-called microphonics, may require an increase in coupling of the cavity with the feeding line in order to increase the cavity bandwidth, and thus to decrease the loaded $Q$ and as a consequence increase the input power necessary to maintain the acceleration gradient. There are two strategies that may be used in order to confront this problem [5]. The first is to apply an active correction of the cavity dimensions by means of a piezoelectric (or magnetostrictive) frequency tuner [6,7]. This piezoelectric tuner changes the cavity length such a way that the detuning caused by microphonics is nearly-perfectly compensated. Due to the partially statistical nature of microphonics noise, a negative feedback loop is necessary. The device must allow correction of micron-scale cavity deformations at frequencies up to about $100 \mathrm{~Hz}$. The piezoelectric tuner allows one to maintain a cavity's resonance frequency by applying a correction directly to the source of the perturbing influence, i.e., to counteract variations in the cavity length. However the piezoelectric tuner requires operation at cryogenic temperatures and thus permits only limited access inside the cryomodule in the event of a failure. Further, the piezoelectric device has its own mechanical resonances which may interfere with control system performance if the self-resonance frequency overlaps with the microphonic excitation to be controlled [5].

A second means for neutralizing microphonics is to use an external tuner to apply a corrective phase shift to the reflected RF wave and reintroduce it to the cavity structure $[8,9]$. Early on, ferrite tuners were suggested for this application [10,11,12]. High-power fast ferrite tuners are currently being developed at CERN for the Superconducting Proton Linac [9] operating at $352 \mathrm{MHz}$. This CERN ferrite tuner is designed to provide fast phase and amplitude modulation of the drive signal for individual superconducting cavities. The tuner is based on two fast and compact high-power ferrite phase shifters magnetically biased by external coils. The tuning frequency for this device can have an upper cut-off at $2 \mathrm{kHz}$ that comes mainly from eddy currents inside the RF structure [11]. 


\section{Omega-P, Inc.}

Final report to DoE on Phase II SBIR grant DE-FG02-06 ER 84472

FAST FERROELECTRIC L-BAND TUNER FOR ILC CAVITIES

The Omega-P SBIR program has as its goal the design of a fast electrically-controlled Lband tuner with a ferroelectric phase shifter [12] that has parameters suitable for ILC application. The tuner will compensate phase errors in real time so as to provide the required phase stability. In addition, this tuner is to allow programmed coupling changes during the cavity filling process that can provide significant AC power savings of about $8 \mathrm{MW}$, or $4 \%$ of the entire power consumption.

As mentioned, the Omega-P design uses electrically-controlled ferroelectric elements to provide the fast tuning. Ferroelectrics have an E-field-dependent dielectric permittivity $\varepsilon(\mathbf{E})$ that can be very rapidly altered by application of a bias voltage pulse. The switching time in most instances would be limited by the response time of the external electronic circuit that generates and transmits the high-voltage pulse, and can therefore be in the nsec range. Euclid Concepts, LLC developed for Omega-P, and tested as part of the Phase I part of the project, a modified bulk ferroelectric based on a composition of BST ceramics, magnesium compounds, and rareearth metal oxides that has a permittivity $\varepsilon=500-550$, and $10 \%$ change in permittivity for a bias electric field of $25 \mathrm{kV} / \mathrm{cm}$. The loss tangent already achieved for the regular samples is less than $6-7 \times 10^{-4}$ at $1.3 \mathrm{GHz}$ (recent preliminary measurements at $1.3 \mathrm{GHz},[14]$ ). The technology of the ferroelectric metallization of large rings $(100 \mathrm{~mm}$ in diameter) is being developed that allows intrinsic switching time less $10 \mathrm{nsec}$ [15]. The availability of this ferroelectric already allows one to create a L-band high-power RF phase shifter with a peak power of over $250 \mathrm{~kW}$ and average power of $4 \mathrm{~kW}$ that necessary for ILC applications.

\section{TECHNICAL APPROACH}

The RF power $P_{g}$ required to maintain an accelerating voltage $V$ is determined by wellknown formula describing the RF cavity excitation by external RF source in the presence of beam loading (see, for example [19]):

$$
P_{g}=\frac{V^{2}(1+\beta)^{2}}{4 \beta Q_{0}(r / Q)}\left[\left(1+\frac{I_{\mathrm{Re}}(r / Q) Q_{0}}{V(1+\beta)}\right)^{2}+\left(\frac{Q_{0}}{1+\beta}\left(\frac{\omega_{0}}{\omega}-\frac{\omega}{\omega_{0}}\right)-\frac{I_{\mathrm{Im}}(r / Q) Q_{0}}{V(1+\beta)}\right)^{2}\right],
$$

where $\omega_{0}$ is the cavity resonance frequency; $Q_{0}$ is it's unloaded quality factor; $\beta$ is the coupling factor, for SC cavity $\beta>>1 ; r / Q$ is the cavity impedance; $I_{R e}=I\left(\cos \delta \varphi_{a}-\cos \delta \varphi_{d}\right), I_{I m}=I\left(\sin \delta \varphi_{a}\right.$ - $\left.\sin \delta \varphi_{d}\right), \delta \varphi_{a}$ and $\delta \varphi_{d}$, are the average phases of the accelerating and decelerating beams compared with the RF phase, respectively; and $I$ is the beam current. For the cooler linac having two cavities with $Q_{o} \approx 4.5 \times 10^{10} @ 2^{\circ} \mathrm{K}$ and $r / Q=404 \mathrm{Ohms} /$ cavity, $I=50 \mathrm{~mA} \times 2=100 \mathrm{~mA}$ and $V \approx 25 \mathrm{MV}$ (the beams will go through the linac twice) [2,20]. The value $\delta \omega=\omega_{0}-\omega$ is determined by the amplitude of uncontrolled noise. The intrinsic RF power required is very small. For parameters listed above, it is 


\section{Omega-P, Inc.}

Final report to DoE on Phase II SBIR grant DE-FG02-06 ER 84472

FAST FERROELECTRIC L-BAND TUNER FOR ILC CAVITIES

$$
P_{\text {int }}=\frac{V^{2}}{Q_{0}(r / Q)}=9 \mathrm{~W} .
$$

If the accelerated and decelerated beams are well balanced, and the beams are in phase with the $\mathrm{RF}$ field, the required power is determined by the peak frequency variations caused by microphonics [21], namely

$$
P_{g}=\frac{V^{2}}{4 Q_{l}(r / Q)}\left[1+\left(2 Q_{l} \frac{\delta \omega}{\omega}\right)^{2}\right],
$$

where $Q_{l}$ is loaded quality factor, $Q_{l}=Q_{0} /(1+\beta)$. From (3) one can find the optimal value of the loaded quality factor, $Q_{\text {opt }}=\omega / 2 \delta \omega$, and minimal required power $P_{g}$ is proportional the peak cavity detuning, namely

$$
P_{g}=\frac{V^{2}}{(r / Q)} \frac{\delta \omega}{\omega}=0.55 \mathrm{~kW} \times d f[\mathrm{~Hz}]
$$

where $\delta f=\delta \omega / 2 \pi$, the peak microphonic cavity detuning in Hz. If, for example, the peak cavity detuning is reduced to $30 \mathrm{~Hz}$ (a typical value), the required input power is $\sim 17 \mathrm{~kW}$ for four 5 -cell cavities. While beam loss within reasonable limits gives no significant increase in required power, the phase error $\delta \varphi$ of the beams does, because in this case the beam introduces an additional reactance proportional to $\delta \varphi$ as it can be seen from (1). The required power in this case $P_{g}$ is equal to

$$
P_{g}=\frac{V^{2}}{(r / Q)}\left(\frac{\delta \omega}{\omega}+\frac{(r / Q) I \delta \varphi}{2 V}\right)=0.55 \mathrm{~kW} \times d f[\mathrm{~Hz}]+22 \mathrm{~kW} \times \delta \varphi\left[^{\circ}\right] .
$$

For, for example, $\delta \varphi=1^{\circ}$ and $\delta f=30 \mathrm{~Hz}$, the required power is about $40 \mathrm{~kW}$. The optimal loaded quality factor in this case is $10^{7}$, a very modest value [22]. Of course, proper cavity detuning is necessary to compensate the reactance introduced by the beam and minimize the required power. Note that for $Q_{l}$ significantly higher than $10^{8}$, there may be problems with field startup, because the cavity bandwidth will be very small compared to the Lorentz-force detuning [22]. Another problem for higher $Q_{l}$ may be with low level RF control that should provide accurate compensation of any perturbation of the small-bandwidth cavity resonant frequency [22,23]. Thus, the cavity resonant frequency control and control of the coupling of the cavity with the feeding line reduces the power demands and provides stable operation of the SRF linac. Note, that in [2] the $Q_{l}$ that is discussed is $3 \times 10^{6}$, that corresponds to a bandwidth of $233 \mathrm{~Hz}$, but requires a power of $130 \mathrm{~kW}$ for all four cavities, or $32.5 \mathrm{~kW} /$ cavity.

The approach considered here is to use an external tuner in order to control the phase and amplitude of fields in the accelerating cavity. In Fig. 2 the standard configuration is shown, where the RF source output is coupled to the SRF cavity through a protection system that 


\section{Omega-P, Inc.}

Final report to DoE on Phase II SBIR grant DE-FG02-06 ER 84472

FAST FERROELECTRIC L-BAND TUNER FOR ILC CAVITIES

includes a circulator and load. All the reflected power from the cavity is fed to the load port of the circulator. This configuration is planned to be used at the Cornell ERL [21]. Needless to say, a fast piezoelectric tuner internal to the cavities is necessary in this configuration.

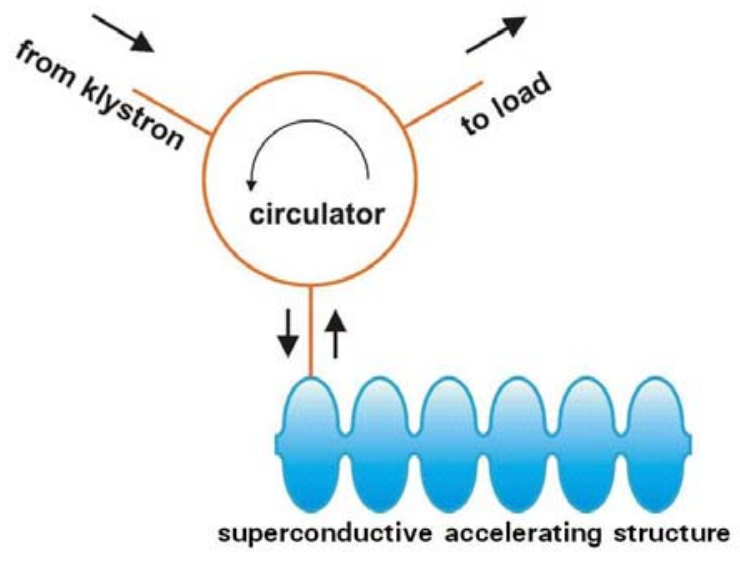

Fig. 2. Standard configuration for connecting RF source to accelerator structure.

In Fig. 3 an alternative arrangement that includes an external tuner in a magic-T configuration that, by use of a feedback loop, adjusts phase and amplitude of fields in the cavity. This configuration is widely used for superconducting cavity control $[10,11,12]$ and requires two fast electrically-controlled phase shifters. The total power from the RF source that is considered is $50 \mathrm{~kW} /$ cavity [26] to be on the conservative side. The phase shifter should provide a phase shift up to $\pm 90^{\circ}$, phase resolution better than $\pm 1^{\circ}$ and very low losses $(<0.7-0.8 \mathrm{~dB})[5,12,25]$. This arrangement employs a magic- $\mathrm{T}$ with two coaxial phase shifters containing ferroelectric elements, as shown in Fig. 5, and allows fast electrically-controlled coupling and phase changes. A magic-T with two phase shifters can provide independent changes of both amplitude and phase of the transmitted wave. Changing the phase from $-90^{\circ}$ to $+90^{\circ}$ will change the tuner transmission from 0 to 1 , or the phase from $-90^{\circ}$ to $+90^{\circ}$ [27]. For a symmetric magic- $\mathrm{T}$, the power level at each phase shifter is only half of the total power incident in the input port, which thereby reduces the temperature rise in the phase shifter active element (ferroelectric) that must be accounted for in the design. This device need not be under vacuum, and is to be located outside of the cryostat.

The phase shifter described early in this program is a coaxial line containing a half-wave ferroelectric ring [27] with matching linear ceramic elements and terminated by a coaxial resonator, as shown in Fig. 6. Applying bias voltage between the central and outer conductors of the coaxial line effects a change in dielectric permittivity of the ferroelectric ring, which causes a phase advance of the RF wave in the phase shifter, and thus a change in coupling between the cavity and the RF source. The matching alumina rings serve to decrease the electric field in the ferroelectric ring. The end capacitor allows one to apply bias voltage to the central electrode. 


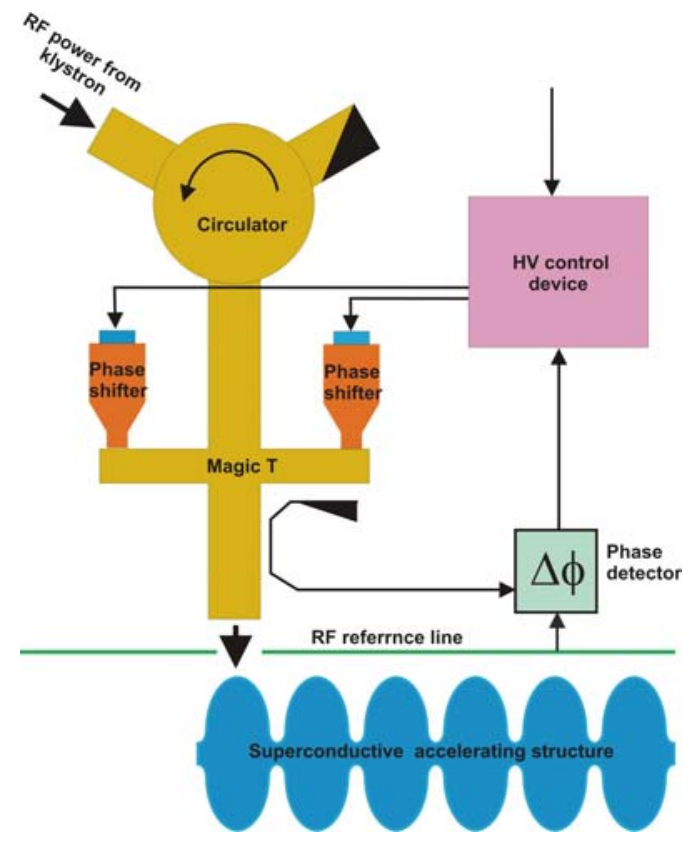

Fig. 3. A possible configuration of the SRF cavity phase and amplitude control using an external tuner in a magic-T configuration with adjustable phase shifters.

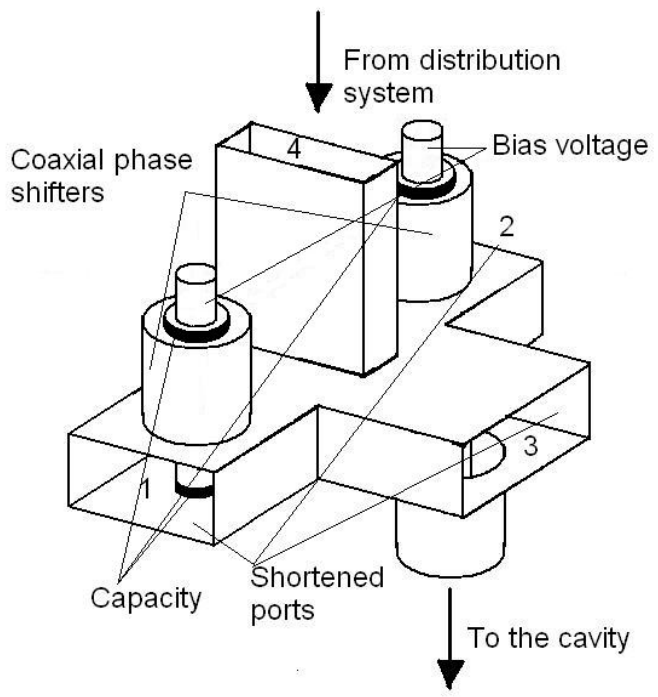

Fig. 4. Schematic of an arrangement to produce fast cavity coupling changes based on a magic-T and two phase shifters containing ferroelectric elements [27]. 


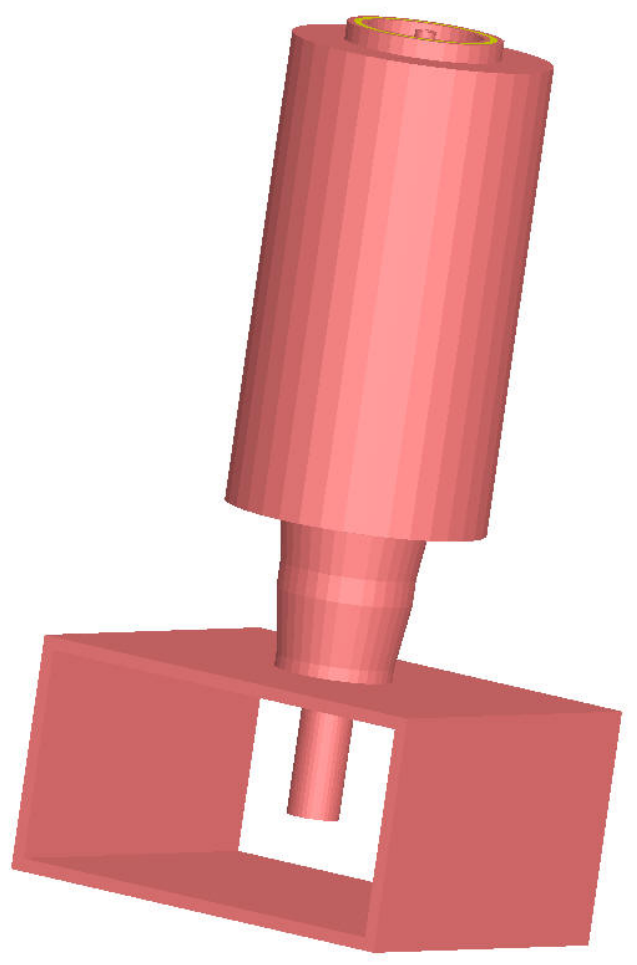

Fig. 5a. Coaxial ferroelectric tuner coupled into rectangular waveguide

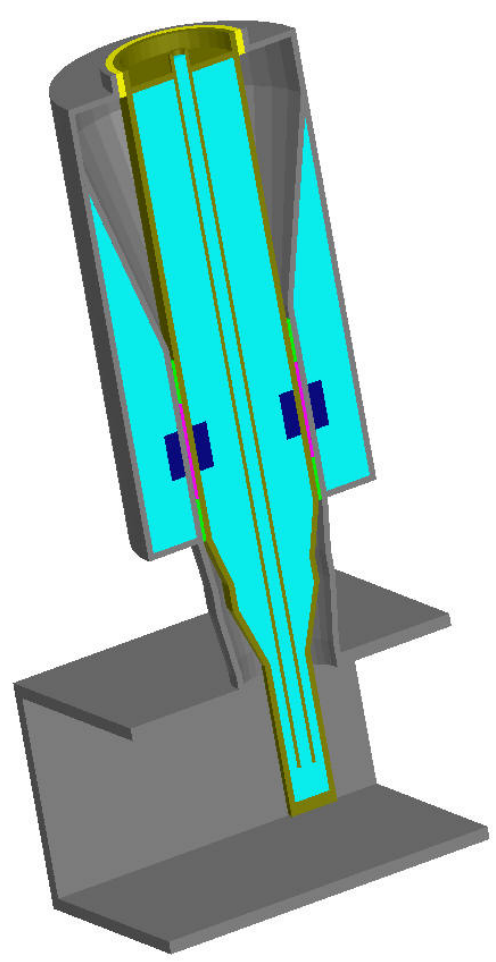

Fig. 5b. Cutaway view of coaxial tuner, showing impedance transformer and ferroelectric and alumina rings.

Considerable analysis showed that, in principle, a coaxial design could satisfy the requirements outlined above. However it soon became apparent that fabrication of the coaxial tuner would be difficult and costly. The insulated central conductor is supported at the upper high-voltage feed-through and further down at the ferroelectric and alumina rings. For good electrical and mechanical support, the rings should be brazed or tightly clamped between the inner and outer coaxial conductors. Design of this braze would need to take into account the greatly differing thermal expansion coefficients for the elements of the coaxial line. Suffice it to say that at this stage a reliable means of brazing ferroelectric rings is progressing, but is not yet reliable enough to bank on for a delicate application such as this. Nor has a means for solidly clamping coaxial elements against one another so far emerged. Consequently, as shall be described in Section III, a radically new concept for the tuner was devised in which solid clamping is straightforward, and in which brazing is probably not an unrealistic option. 


\section{PLANAR GEOMETRY FERROELECTRIC TUNER CONCEPT}

In view of the aforementioned difficulty in conceiving of a practical means for brazing and/or firmly clamping a ferroelectric ring and necessary matching ceramic rings between coaxial conductors, a planar geometry was investigated as an alternative. In this geometry, all elements of the design (conductors, ferroelectric elements, and matching ceramic elements) are planar. Thus rigid clamping of the structure is straightforward. Furthermore, since stress need be applied along only one axis of the system (as opposed to two in cylindrical geometry), it is planned to use a low-melting alloy braze material to effect a solid joint between the metallic conductors and both types of insulators.

Drawings are shown in Figs. 6 to illustrate the new arrangement for the tuner. As is seen, it consists of a "three-decker club sandwich" clamped vertically within an L-band rectangular waveguide. For clarity, two high-voltage feed-thrus on side walls of the waveguide that connect to the electrically-insulated pink electrodes for imposition of bias fields are not shown. The sandwich arrangement allows good electrical contact between metallic, ferroelectric, and ceramic elements by use of vertical clamping; allows use of lower voltages since the ferroelectric bars are only about one-third the height needed in a single-deck sandwich, and should allow a lowtemperature braze to assure good electrical contact between metal, ferroelectrics, and ceramics.

Ferroelectric and ceramic bars and rods for the planar geometry tuner are developed and supplied to Omega-P by Euclid TechLabs LLC. Properties of the ferroelectrics are listed below $[27,30]$. Analysis and simulations for the tuner are based on use of these properties.

TABLE I. Properties of ferroelectric ceramics

\begin{tabular}{|c|c|}
\hline dielectric constant, $\varepsilon$ & $\sim 550$ \\
tunability, $\partial \varepsilon / \partial E_{\text {bias }}\left(E_{\text {bias }}\right.$ is the bias field) & $>2 /\left(\mathrm{kV} \cdot \mathrm{cm}^{-1}\right)$ \\
response time & $<10 \mathrm{~ns}$ \\
loss tangent at $1.3 \mathrm{GHz}, \delta$ (preliminary measurements) & $\sim 6.5 \times 10^{-4}$ \\
loss tangent at $0.7 \mathrm{GHz}, \delta$ (estimated)* & $\sim 3.5 \times 10^{-4}$ \\
breakdown limit & $200 \mathrm{kV} / \mathrm{cm}$ \\
thermal conductivity, $K$ & $7.02 \mathrm{~W} / \mathrm{m}-\mathrm{K}$ \\
specific heat, $C$ & $0.605 \mathrm{~kJ} / \mathrm{kg}-\mathrm{K}$ \\
density, $\rho$ & $4.86 \mathrm{~g} / \mathrm{cm}^{3}$ \\
coefficient of thermal expansion & $10.1 \times 10^{-6} \mathrm{~K}^{-1}$ \\
temperature tolerance, $\partial \varepsilon \partial \mathrm{T}$ & $3 \mathrm{~K}^{-1}$ \\
\hline
\end{tabular}

*We assumed a very modest (pessimistic) value of $5 \times 10^{-4}$ for preliminary considerations. Note that the final design will be based on measurements of loss tangent that are in progress at Omega-P. 


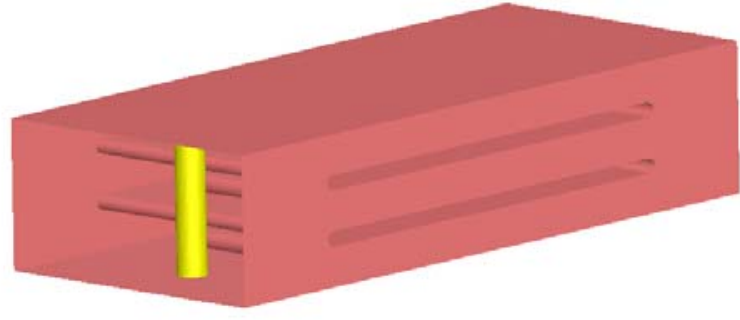

Fig. 6a. Rectangular L-band waveguide tuner, within which the three-decker club sandwich assembly is housed.

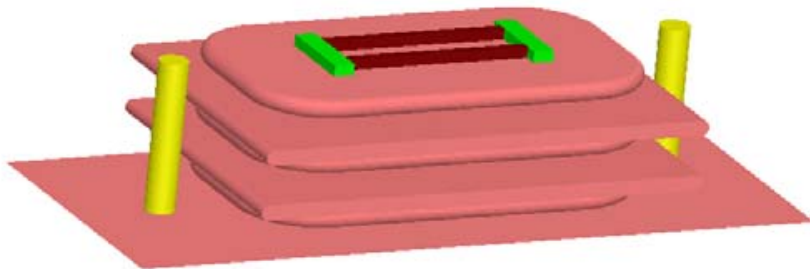

Fig. 6c. Perspective view of three-decker club sandwich with waveguide top and side walls removed, showing one set of ferroelectric and matching bars (dark red and green), and the vertical ceramic matching rods (yellow).

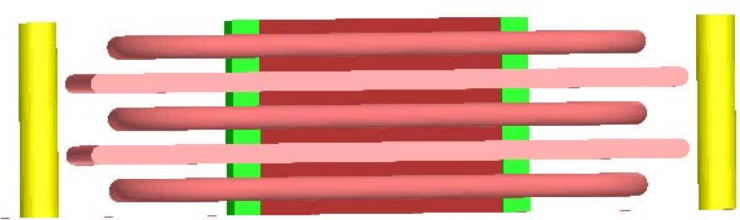

Fig. 6b. Side view of three decker club sandwich. Ceramic matching rods are yellow and bars are green, ferroelectric bars are dark red, grounded electrodes are light red, and HV biased electrodes are pink.

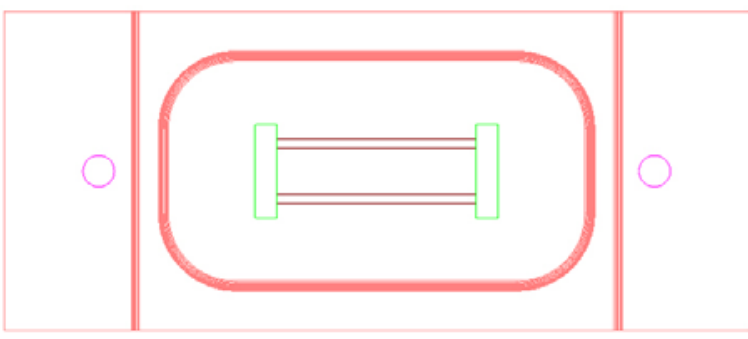

Fig. 6d. Layout seen from above of one of the layers within the three-decker sandwich.

Oval outlines the HV biased electrode, vertical lines outline the grounded electrode, green outlines the matching ceramic bars, red circles are the vertical maching ceramic rods, and horizontal violet lines outline the ferroelectric bars.

\section{IIIa. Simulation of tuner performance.}

A low-power single-decker mockup version of the tuner was built and tested, scaled to operate at $1300 \mathrm{MHz}$. Fig. 7 illustrates the geometry for the simulations, corresponding to onesixth of the three-decker sandwich, while Figs. 8 and 9 show results. Fig. 10 shows dimensions calculated for a $1.3 \mathrm{GHz}$ version of the structure.

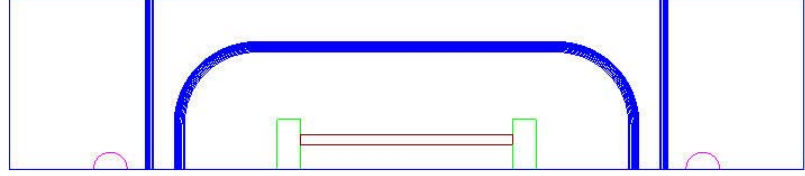

Fig. 7a. Top view of geometry for simulation.

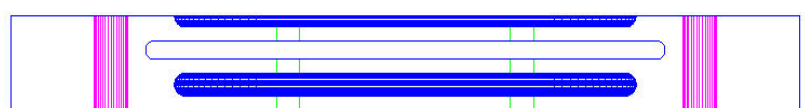

Fig. 7b. Side view of geometry for simulation. 


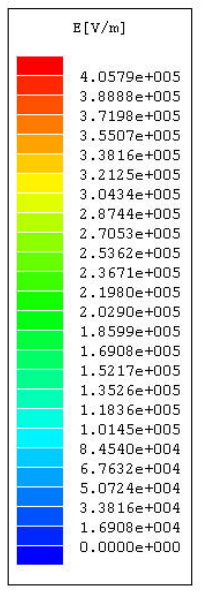

Eps $=470$

Fig. 8. Electric field distribution surrounding ferroelectric and ceramic elements for $\varepsilon=470$ for the ferroelectric elements. Patterns for other nearby values of $\varepsilon$ are similar.
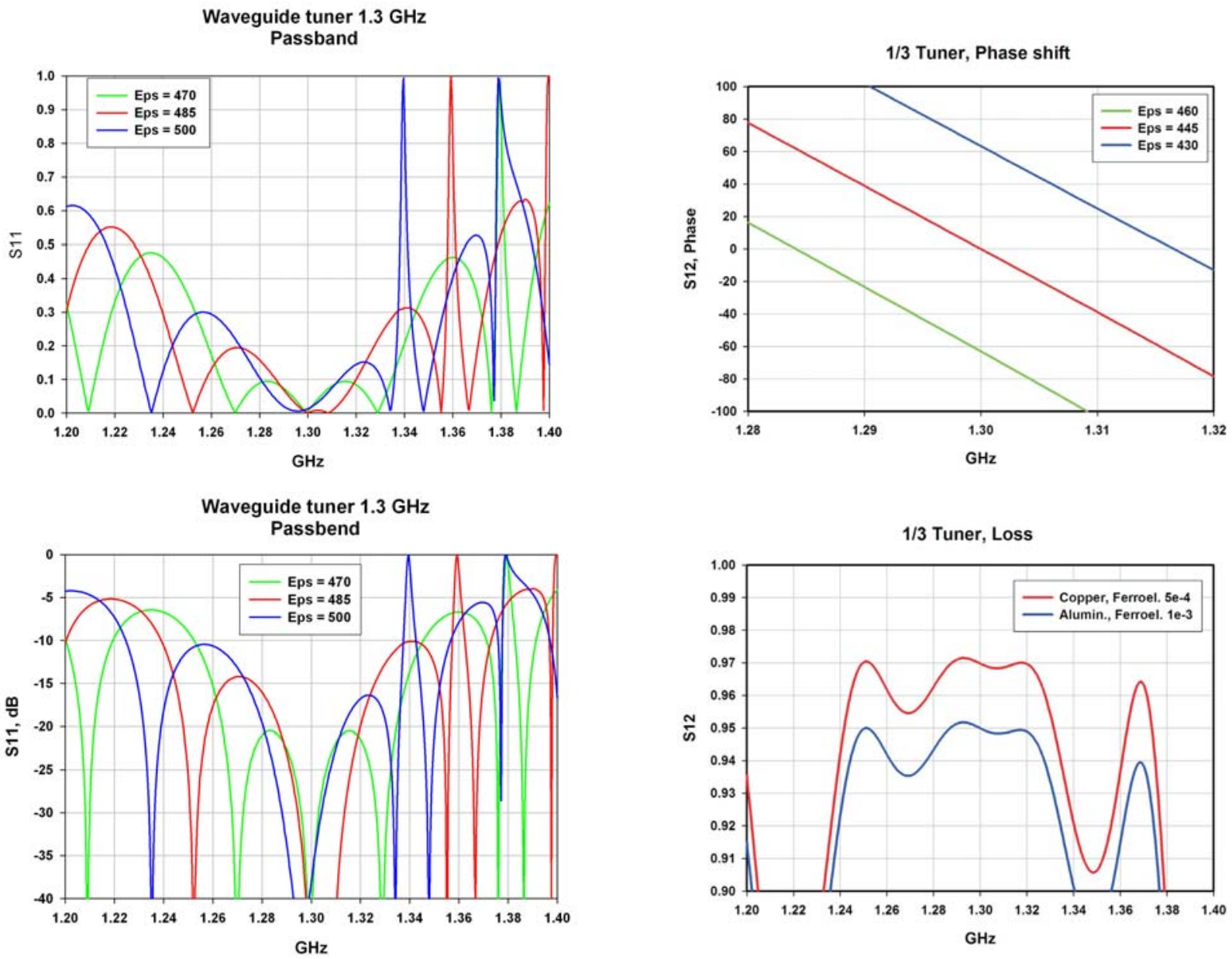

Fig. 9. Calculated pass band, phase shift, and loss for the $1.3 \mathrm{GHz}$ tuner for three values of $\varepsilon$. 

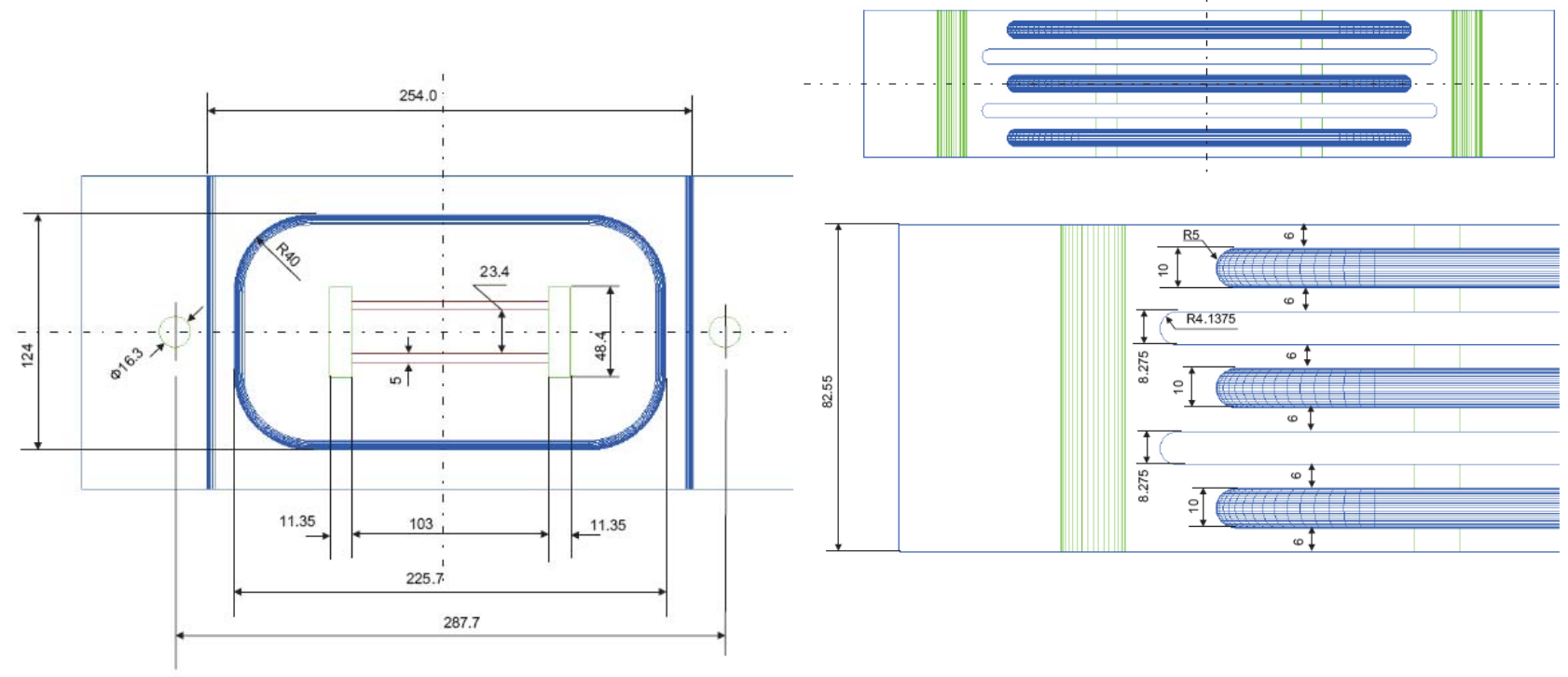

Fig. 10. Layout of triple-decker $1300 \mathrm{MHz}$ tuner structure with dimensions (in $\mathrm{mm}$ ).
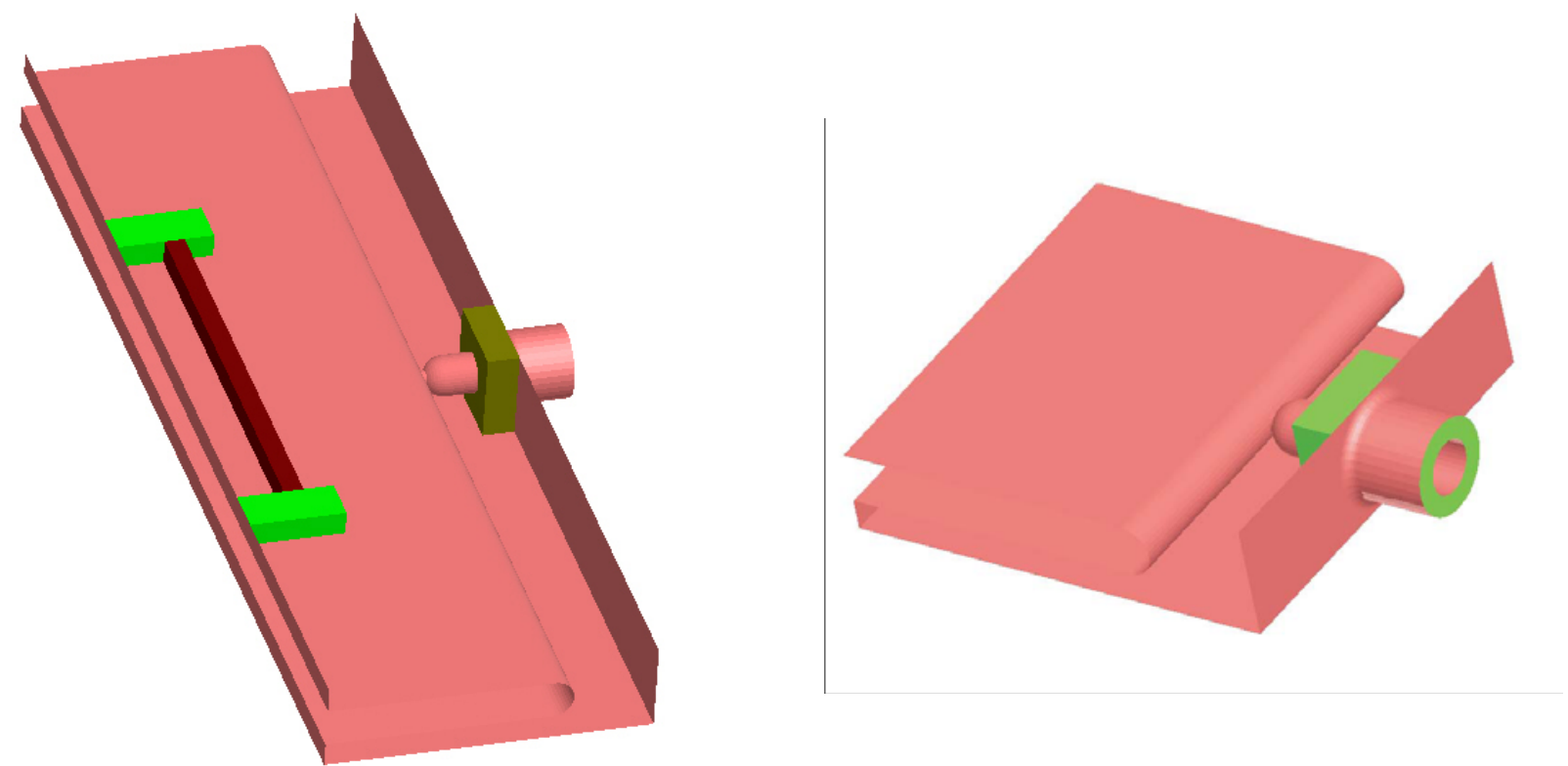

Fig. 11. Detail of high-voltage feed-thru, one of two in the triple-decker tuner. 


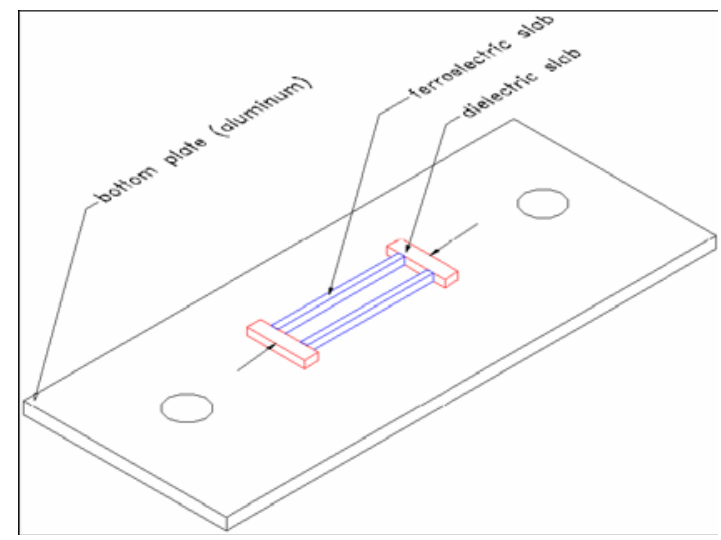

Step 1: Install bars on bottom plate.

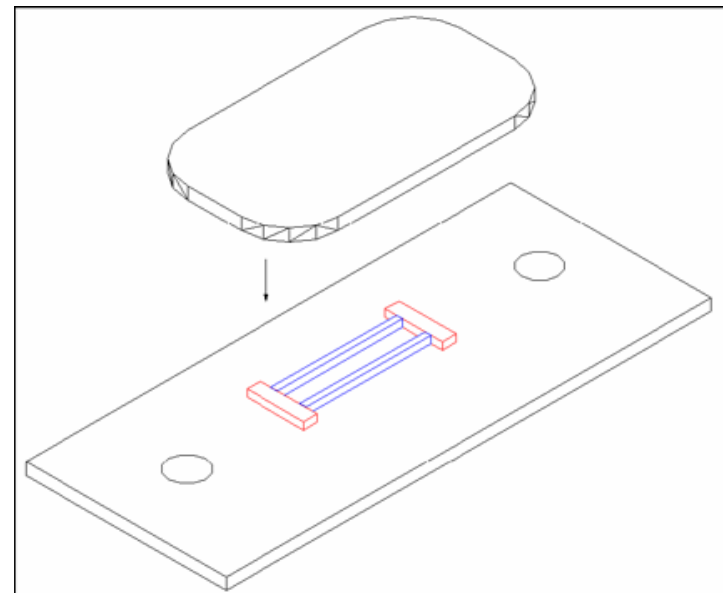

Step 2: Install bias plate on top of bars.

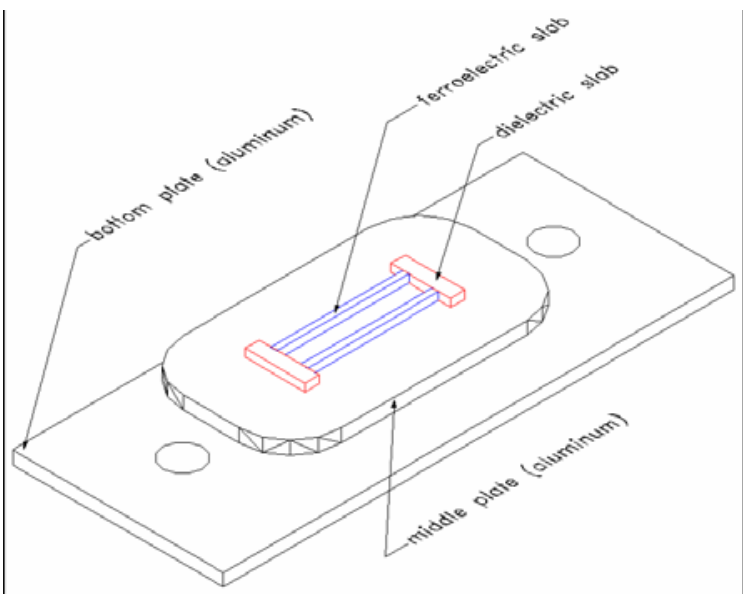

Step 3: Install second set of bars.

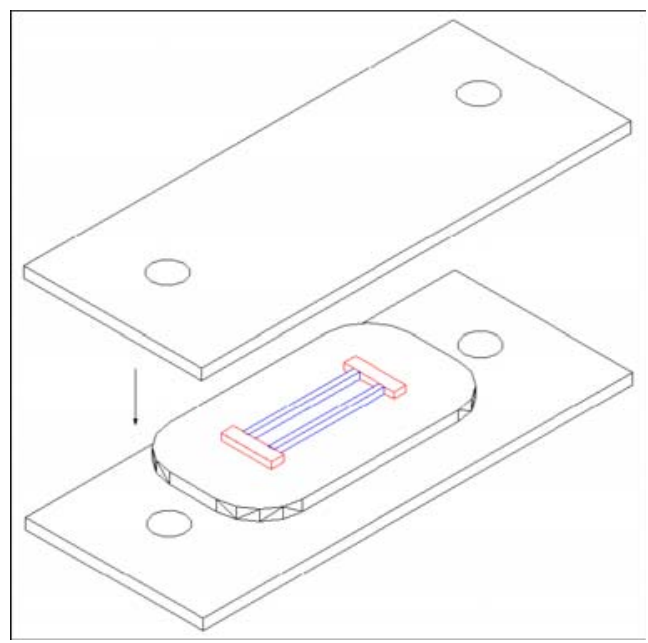

Step 4: Install top plate.

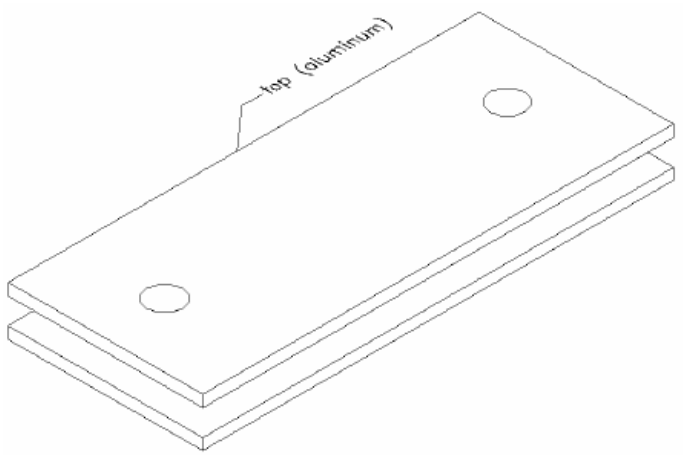

Step 5: Secure and clamp top plate.

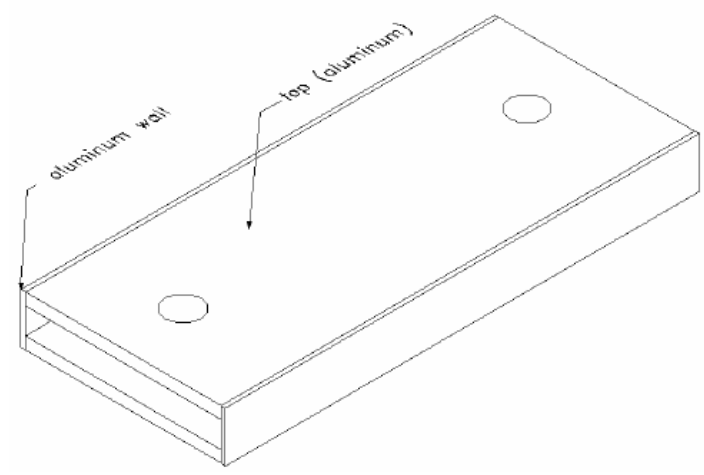

Step 6: Attach waveguide side walls.

Fig.12.. Assembly steps for one-third model tuner structure. 


\section{IIIb. Measurements of loss tangent of ferroelectric material}

In view of the critical influence that losses in the ferroelectric elements have upon performance of the tuner, it was necessary to develop a simple means to measure the loss tangent for each sample supplied by Euclid TechLabs. The method used is based on excitation of a coaxial mode in a cylindrical conducting pipe within which the sample is suspended, as seen in Fig. 13. Coupling of power between the RF loops shows a resonance that depends on the specific geometric arrangement, but where losses in the ferroelectric are dominant. Fortunately, for the bars used in the tuner, one resonance is found at about $1400 \mathrm{MHz}$, close enough to $1300 \mathrm{MHz}$ for the measured value to be relevant to operation of the tuner at 1300 MHz. A typical resonance is shown in Fig. 14. For this coaxial mode, the measured $Q$ value is 1620. Now, since

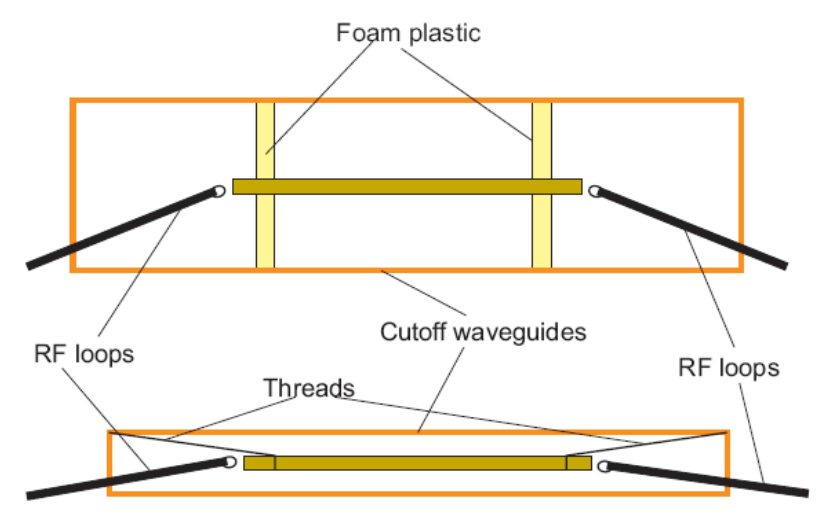

Fig. 13. Arrangement for measurement of loss tangent in ferroelectric bar.

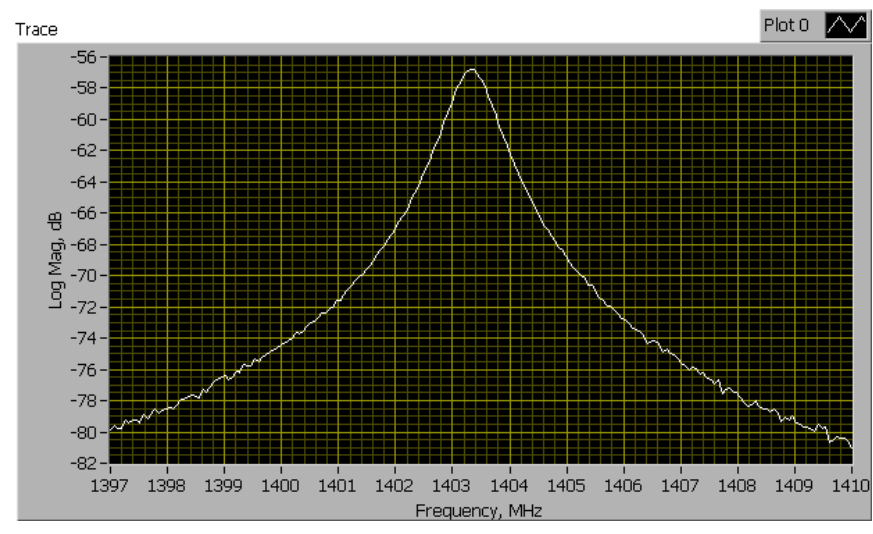

Fig. 14. Resonance curve for loss tangent measurement.

HFSS analysis for the arrangement shows the ratio of total energy stored to that stored in the bar is 3.2:1, one can infer that the ferroelectric loss tangent is $3.2 / 1620=2 \times 10^{-3}$. This value is in good agreement with that measured by Euclid for this batch of ferroelectric samples. Unfortunately, as shall be seen, this degree of loss is 2-3 times larger than can be tolerated for the tuner, thereby requiring further development by Euclid. 


\section{Omega-P, Inc.}

Final report submitted to DoE on Phase II SBIR grant DE-FG02-06 ER 84472

FAST FERROELECTRIC L-BAND TUNER FOR ILC CAVITIES

\section{IIIc. Bench tests of one-third model of planar geometry ferroelectric tuner}

Experiments have been carried out on a reduced scale model of the planar geometry ferroelectric tuner. In the model, only one of the three layers in the triple-decker club sandwich is tested. It is assembled as shown in Fig. 12. Moreover, for convenience, the model is scaled up in frequency to $1300 \mathrm{MHz}$, and thus it is roughly half the size of a $700 \mathrm{MHz}$ version. Tests at $1300 \mathrm{MHz}$ also allow the results to be judged with respect to applications for ILC and other accelerator projects that operate at this frequency. A photograph of the experimental set-up in the Yale University Beam Physics Laboratory is shown in Fig. 15.

Fig. 15 shows the clamped one-third scale $1300 \mathrm{MHz}$ tuner assembly at the center joined by two tuned tapers to standard WR-650 coax-to-waveguide adaptors, thence by coax cables to the vector network analyzer. DC voltages up to about $9 \mathrm{kV}$ were applied to the center insulated plate of the structure with the two cables from the power supply enclosed in the plastic box at the right. Measurements were made of transmission and reflection versus frequency, for various values of applied DC voltage.

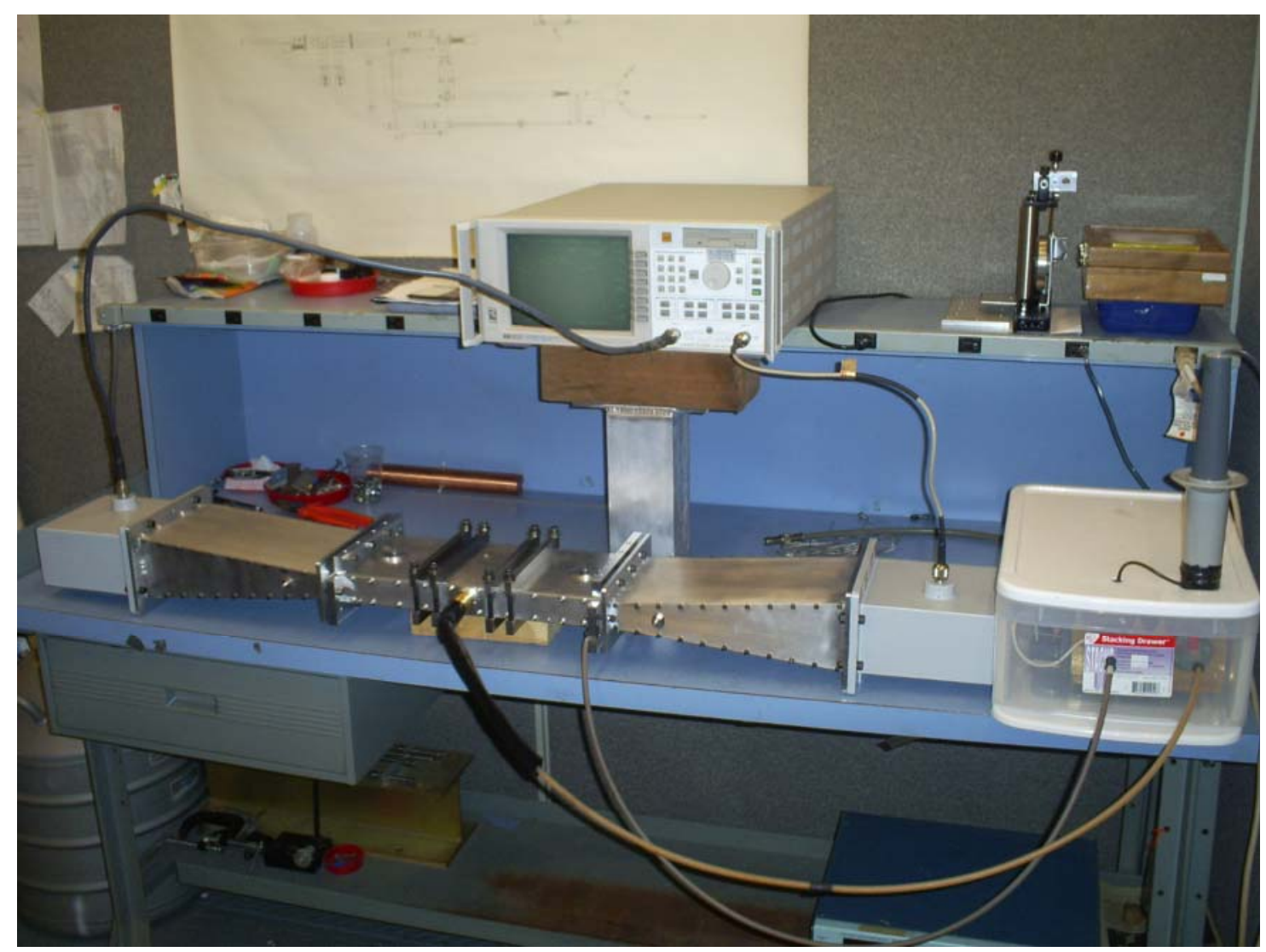

Fig. 15. Test set-up in the Yale Beam Physics Laboratory for one-third version of tuner. Note waveguide tapers from full height to reduced height of test section, and high-voltage bias supply (at right). 
For the first series of tests, ferroelectric and ceramic bars were installed "dry," which is to say without any material to insure a tight fit adjacent to the conducting surfaces. Application of high-voltage led to arcing that made experiments impossible. Bars with gold metallization were prepared and the surfaces of the bars that make contact with the conductors were coated with a liquid metal (indium-gallium alloy) before installation. This allowed voltages up to 9 $\mathrm{kV}$ to be applied without serious arcing. Phase shift measurements, with dielectric constant $\varepsilon$ varied by application of bias voltage were as follows:

$$
\begin{aligned}
& \varepsilon=460, \text { phase shift }=0 \mathrm{deg}, \\
& \varepsilon=445, \text { phase shift }=63 \mathrm{deg}, \\
& \varepsilon=430, \text { phase shift }=126 \mathrm{deg} .
\end{aligned}
$$

A graph of phase shift versus voltage is shown in Fig. 16. The different curves correspond to dry installation between aluminum electrodes, dry installation between copper electrodes, and "wet" installation between copper electrodes. Here "wet" refers to the use of the liquid metal. As is seen in Fig. 16, the phase shift data are essentially independent of the method of installation.

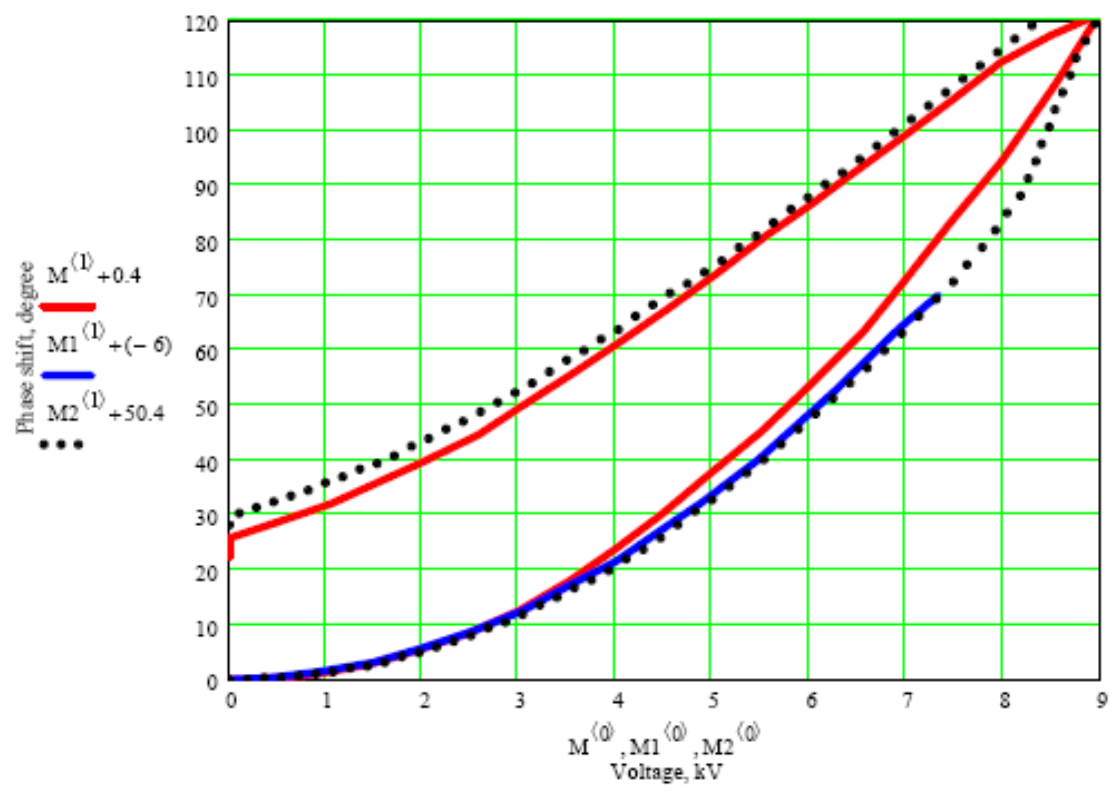

Fig. 16. Measurements of phase shift versus applied voltage for the ferroelectric tuner, where different curves correspond to different methods of installation. (See text for explanation.) A range of phase shift up to $120^{\circ}$ is seen for applied voltages up to $9 \mathrm{kV}$. Characteristic hysteresis is seen, with the lower set of curves for raising voltage and the upper set for falling voltages.

These data establish one vital property of the tuner, namely its electrically-tunable phase shift. But this property should be without serious loss of transmitted power for the tuner to be useful. Results of transmission measurements for this arrangement are shown in Fig. 17, taken for the installation of the bars with liquid metal and copper electrodes. 


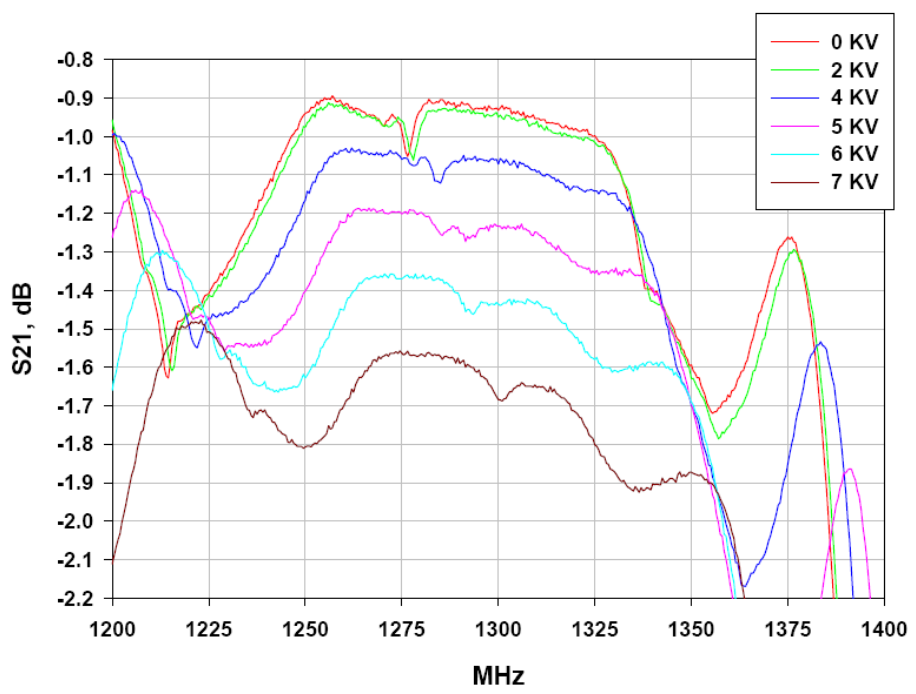

Fig. 17. Transmission through the ferroelectric tuner vs frequency, for several applied voltages.

The data shown in Fig. 17 exhibit two disturbing features. The first feature is the degree of transmission loss, namely $0.93 \mathrm{~dB}$ at $1300 \mathrm{MHz}$, even in the absence of applied voltage. This corresponds to a $20 \%$ loss - clearly too high for most applications. The second feature is the dependence of loss upon applied voltage, amounting to as much as $1.7 \mathrm{~dB}$ for the $7 \mathrm{kV}$ case, a $32 \%$ loss of transmitted power. It should be stated that reflection losses are much smaller for this arrangement. There is no record in the literature of ferroelectric materials that shows the loss to depend upon applied electric field. Thus, one is led to conclude that this unusual behavior is probably due to properties of the liquid metal used. Furthermore, a calculation for the measured loss tangent of $2 \times 10^{-3}$ indicates that the transmission loss should be only $14 \%$, and not $20 \%$. So even with no applied voltage, an anomalous loss appears to be present.

To try to eliminate this anomalous loss, ferroelectric and ceramic bars were prepared with gold metallization and installed with 0.010 " indium foil between the bars and the electrodes, to see if the soft indium would, under pressure, flow to fill voids and establish solid electrical contact. This attempt did not materially improve the situation. So the structure, with the indium foil in place and under about 2 tons pressure, was heated to above the indium melting point $\left(156^{\circ} \mathrm{C}\right)$ in an attempt to form a soldered joint. This apparently did occur, as seen in Fig. 18-19. 


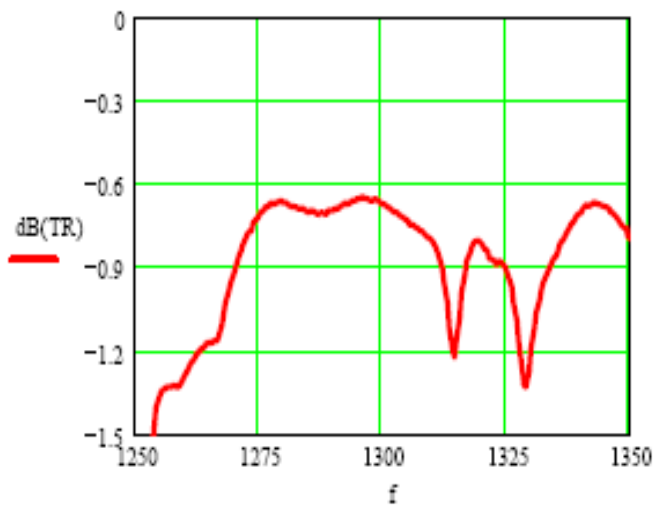

Fig. 18. Transmission coefficient for tuner with "soldered" ferroelectric and ceramic bars.

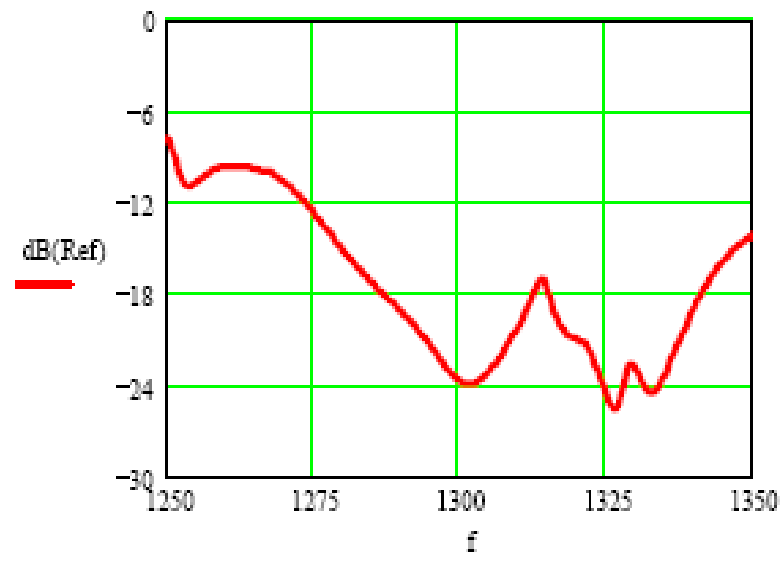

Fig. 19. Reflection coefficient for tuner with "soldered" ferroelectric and ceramic bars.

The measured transmission loss at $1300 \mathrm{MHz}$ of $-0.66 \mathrm{~dB}$ seen in Fig. 18 corresponds to $14 \%$, exactly as was calculated for a ferroelectric loss tangent of $2 \times 10^{-3}$. The measured reflection loss of --24 dB adds another $0.4 \%$--insignificant on this scale. It would of course have been natural to next measure the transmission loss with applied voltage, but unfortunately this could not be done. During the melting of indium under high pressure on the joints, excess molten indium evidently squirted out of the joints and in some places deposited on the sides of the ceramic bars - thereby introducing small short circuits that prevented application of voltage.

But the highly significant point shown by this last measurement is that the predicted RF performance can be observed when the interfaces are filled with indium between the copper electrodes on one hand, and the gold-coated ferroelectric and ceramic bars on the other. Evidently small gaps between the high dielectric constant ferroelectric and the metal surfaces introduce excess loss. Further, one can tentatively conclude, use of liquid metal as a joint filler substance is not a solution, due perhaps to polarization effects. In any case, these last results 
show that a reliable means must be developed for establishing good solder or brazed joints between the dielectrics and metals in order for the ferroelectric tuner to perform satisfactorily.

But even with satisfactory electrical joints between the dielectrics and the metals, the $14 \%$ loss in the ferroelectrics is still at least a factor-of-two too large. Thus, a further goal for Euclid TechLabs for the future is to improve the properties of the ferroelectric, reducing losses without undue reduction in tunability.

\section{IIId. Measurements of tuner loss}

The loss tangent of ferroelectric bars was measured for the uncoated bars (manufactured from the same batch used to make the bars coated with gold), as described above. The value of loss tangent was determined to be $\sim 2 \times 10^{-3}$, suggesting that the $1 / 3$ scaled tuner model would suffer a transmission loss no better than $\sim 0.7 \mathrm{~dB}$. In actuality, the measured transmission was worse, with minimum transmission loss found only when we used either freshly applied liquid InGa or soldered the bars to the waveguide walls (using In). However, we were never been able to apply more than $4 \mathrm{kV}$ to the soldered configurations, probably due to small solder leakage flow along crevices; hence we discuss below only the structures assembled with liquid InGa. It was found that in the configurations with fixed structure height, when the top and bottom walls are tethered by bolting to the side walls, the transmission dropped when the voltage grew [see Fig. 20]. However, in a configuration where the top wall was resting without tethering on the ceramic bars under 200-400 lbs load, the transmission did not drop so much. However, in general, the transmission level was lower because of leakage radiation through the gaps formed between unbolted walls, or in some cases became even higher [see Fig. 21]. This suggests the possible presence of piezoelectric effects that lead to the contraction of the bars and degradation in quality of the bar-wall surfaces contacts. It is our understanding that the losses are partially caused by the bad contact between ceramic and the waveguide walls, and is not an intrinsic property of the phase shifter. It is anticipated that successful brazing of the ferroelectric and matching dielectric bars should eliminate losses above those in bulk ceramics and metallic walls, and lead to transmission being independent of applied voltage. Development of brazing technology is clearly an immediate task for Euclid to undertake immediately, a task that it is committed to pursue.

\section{IIIe. Measurements of tuner switching speed}

A vital property of any tuner is its response time, which for many accelerator applications should be less than $100 \mathrm{~ns}$. This is clearly shorter than what can be achieved with a ferrite tuner, for example. Measurements of response time were made using the arrangement shown in Fig. 22. The high voltage rise/fall times from the available pulse generator were in the range of $\sim 100 \mathrm{~ns}$, measured as the time difference from $5 \%$ to $95 \%$ of the voltage maximum. Results are depicted in Fig. 23. 


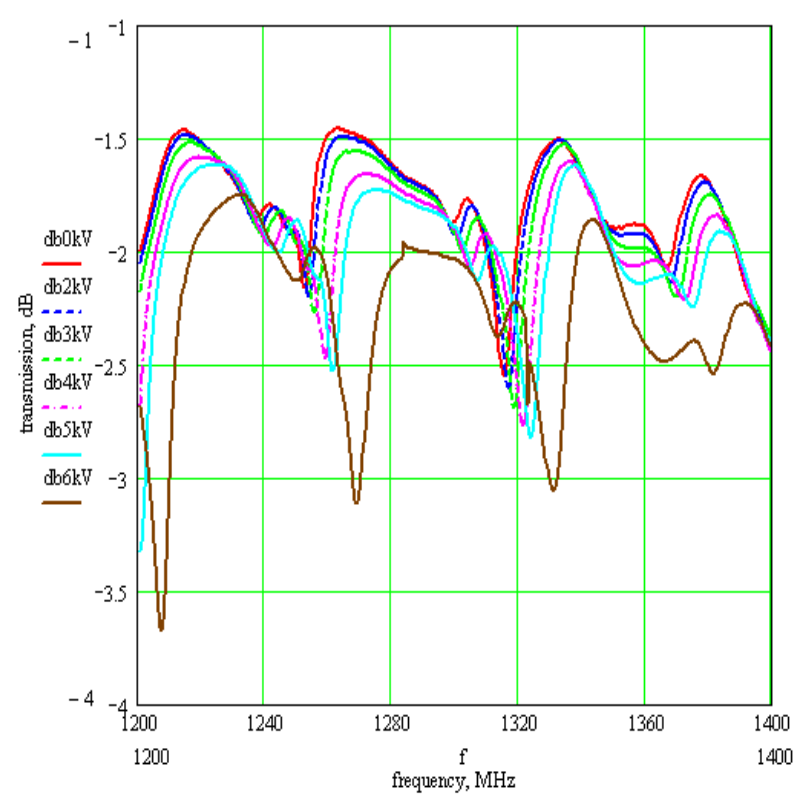

Fig. 20. Transmission drops when voltage is raised in the configuration with fixed structure height (the top and bottom walls are tethered by the side walls), suggesting a piezoelectric contraction of the bars.

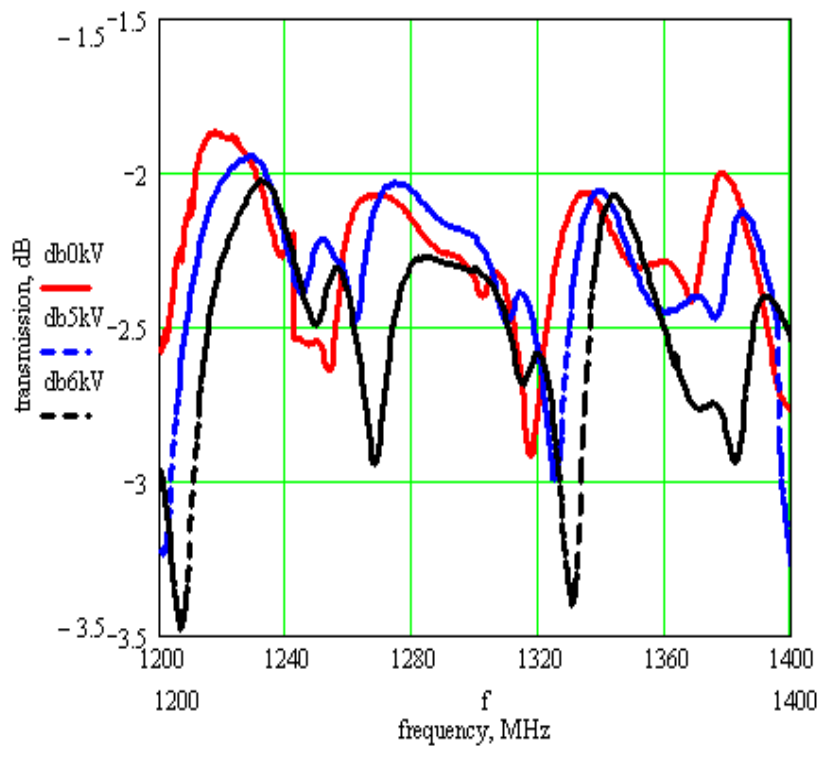

Fig. 21. In the configurations where the top wall was resting under $200-400 \mathrm{lbs}$ load on the ceramic bars, and not bolted to the side walls, transmission did not drop as much as in Fig. 16.

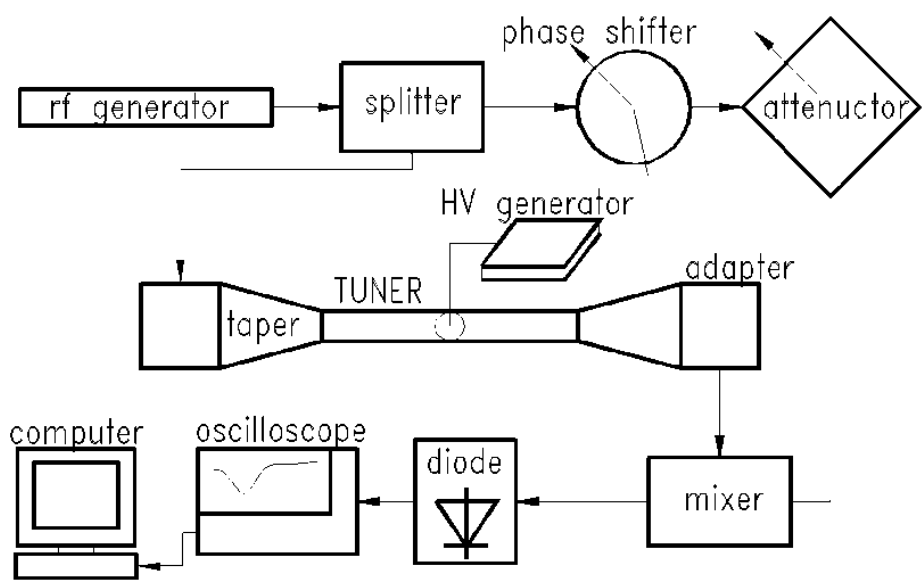

Fig. 22. Arrangement for measurement of tuner switching speed. The signal from the RF generator at $1,290 \mathrm{MHz}$ is split in two. One portion was directed through a phase shifter and attenuator directly to a mixer, while the second portion was fed through the tuner input port, passed through the tuner, picked up at the tuner output port, and then fed to the mixer. The resulting signal from the mixer was detected by a diode and monitored using an oscilloscope, and also captured by a computer for further signal processing (mainly FFT). 


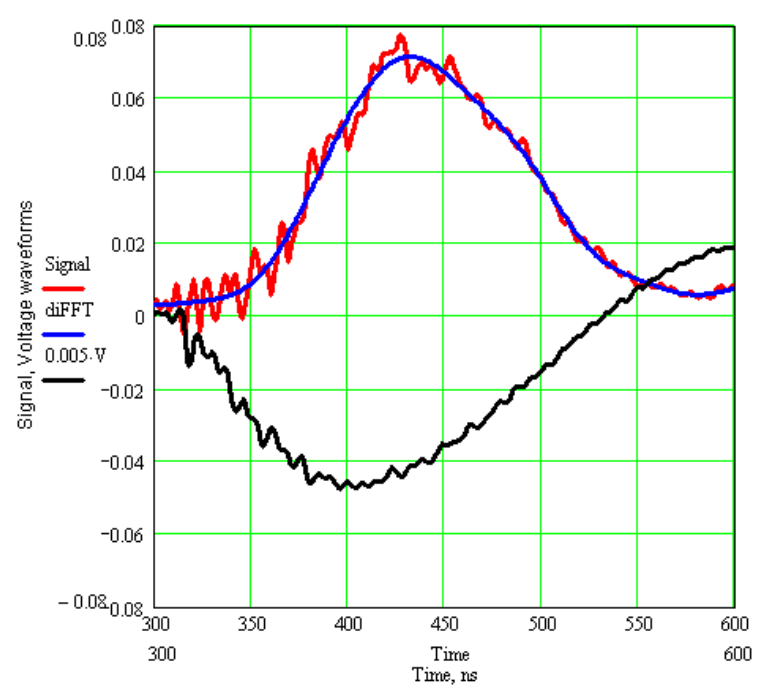

Fig. 23. Time-response of phase shifter. Red curve (convex) is the difference between data with RF off and RF on. Blue curve (also convex) is the processed red curve after direct and inverse FFT with filtering out of high spectral components; the black curve (concave) is the highvoltage pulse. The vertical scale for the latter is reduced by a factor of $2 \times 10^{5}$; its peak value was $9.7 \mathrm{kV}$. It is seen that the time delay between peak voltage and peak variation in phase is $28 \mathrm{~ns}$. (This value excludes delays in cables.) Time scale is 50 ns per division.

Switching speed measurements (each averaged over 16 shots) were processed by subtracting data with RF off from data taken with RF on, as shown in Fig. 23. The difference signal of $67 \mathrm{mV}$ from the mixer corresponds to a phase change of $77^{\circ}$. From these data, where the response time of the phase shifter is dominated by the 90 ns rise time of the voltage pulse, one can infer that the response time to a step function voltage would be equal to or less than the delay time, namely approximately $30 \mathrm{~ns}$. This could be interpreted to correspond to an average switching rate of less than $0.5 \mathrm{~ns}$ for each degree of RF phase. Having shown rapid ( $\sim 30 \mathrm{~ns})$ switching speed, measurements for a $1 / 3$ version of an L-band tuner also have demonstrated phase shift values in good agreement with theory. 


\section{IIIf. Alternative phase shifter concept: a radial line reflector}

An alternative concept for the L-band ferroelectric phase shifter is based on use of a radial line reflector instead of either a coaxial line reflector or the planar sandwich design; the radial line design is depicted in Fig. 24. As can be seen, this design requires metallization on the flat edges of the ferroelectric and alumina rings, but not on the cylindrical surfaces; the former technique is already well developed. Furthermore, assembly of this structure with either brazing or clamping of the rings between the planar surfaces of the two metallic elements would seem to be more straightforward than for the much more complex three-decker sandwich arrangement discussed above. Preliminary analysis of this structure revealed issues with a large number of modes trapped in the rings. Still, it is planned to devote effort to a more detailed analysis of this promising new structure in the future, in view of its mechanical simplicity.

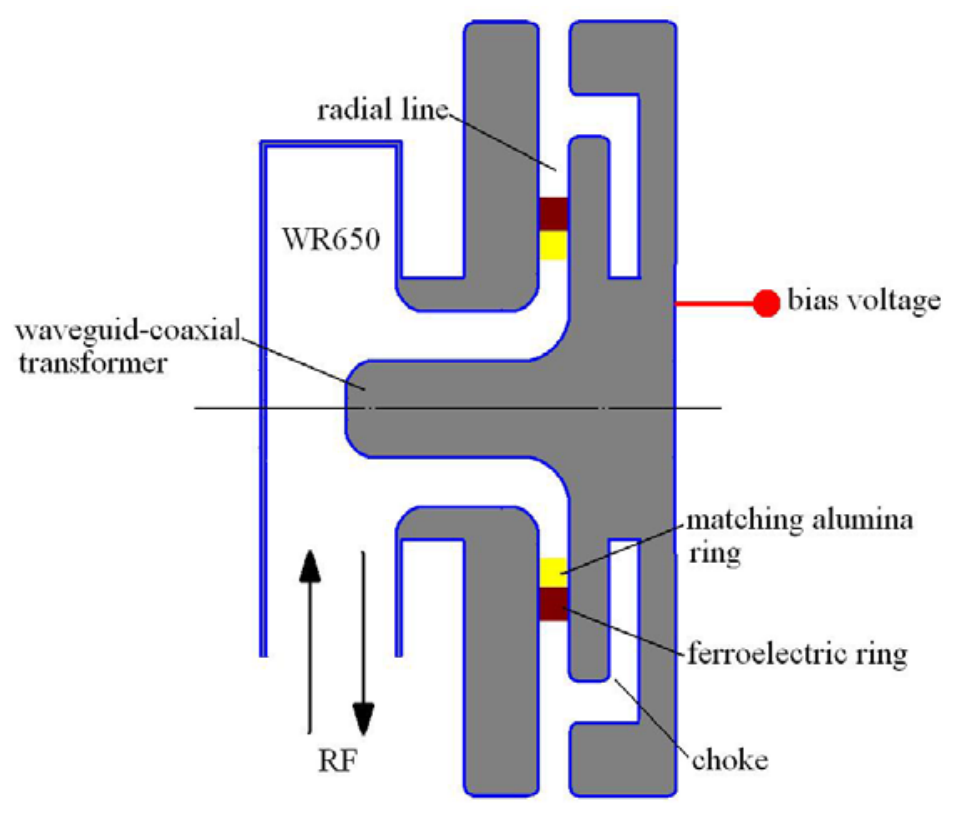

Fig. 24. An alternative phase shifter concept employing a TEM radial line reflector. 


\section{REFERENCES}

1. I. Ben-Zvi, J. Brennan, A. Burrill, et al., "R\&D Towards Cooling Of The RHIC Collider", PAC2003, Portland, May 11-16, 2003, pp. 39-41.

2. I. Ben-Zvi, "The ERL High-Energy Cooler for RHIC", EPAC2006, Edinburgh, 2006, pp. 940-944. See also R. Calaga, "SRF Cavities for High Current ERLs," BNL, January 23, 2006, http://www.bnl.gov/cad/ecooling/docs/PDF/MAC 01 06/calaga.pdf

3. M.M. Karliner, V.M. Petrov, and I.A. Shapiro, "Cavity Wall Instability Caused by Ponderomotive Force of Electromagnetic Field," Soviet Journal of Technical Physics, v. XXXVI, N11, 1966, pp.2017-2027.

4. S. Choroba, "Status of RF System," International Technology Recommendation Panel. ITRP Meeting Two, April 5-6, 2004. DESY - Hamburg, tesla.desy.de/new pages/ham burg meeting 4 2004/talks/ITRP_Choroba_RF.pdf

5. T. Zwart, D. Cheever, W.S. Graves, D. Wang, A. Zolfaghari, "Optimal RF System For Lightly Loaded Superconducting Structures", Proceedings of the 2004 FEL Conference, pp. 542-545; see also web.elettra.trieste.it/fel2004/proceedings/papers/TUPOS57 /TUPOS57.PDF

6. S. Simrock., G. Petrosyan, A. Facco, V. Zvyagintsev, S. Andreoli, and R. Paparella, "First Demonstration of Microphonic Control of a Superconducting Cavity with a Fast Piezoelectric Tuner", PAC2003, Portland, May 11-16, 2003, p.470.

7. M. Liepe and S. Belomestnykh, "Microphonics Detuning in the $500 \mathrm{MHz}$ Supercondicting CESR Cavities," PAC2003, Portland, May 11-16, 2003, pp.1326-1328.

8. Y. Kang, M. Tiefenback, and P. Chevtsov, "Reactive RF Tuning for Compensation of a Detuned Accelerating Cavity," Linac 2002, Gyeongju, Korea, 2002, pp.733-735.

9. Martin Dohlus, DESY, Feb. 2004

10. D. Valuch, H. Frischholz, J. Tückmantel, and C. Weil, "First Results With A Fast Phase And Amplitude Modulator For High Power RF Applications," EPAC2004, pp. 959-961.

11. D. Horan, and E. Cherbak, "Fast-Ferrite Tuner Operation On A 352-MHz Single-Cell RF Cavity At The Advanced Photon Source," PAC2003, pp. 1177-1179.

12. B. Foster, I. Gonin, T. Khabiboulline, A. Makarov, N. Solyak, I. Terechkine, and D. Wildman, "High Power Phase Shifter," PAC2005, PAC2005, Knoxville, May 16-20, 2005, p. 3123-3125.

13. V.P. Yakovlev, S.Yu. Kazakov, and J.L. Hirshfield, "1.3 GHz Electrically-Controlled Fast Ferroelectric Tuner," EPAC2006, Edinburgh, June 26-30, 2006, pp. 487-489.

14. L.C. Sengupta, "Bulk Ceramic Ferroelectrics and Composites: Manufacture, Microwave Properties and Applications," IMS2000; see also http://my.ece.ucsb.edu/yorklab/Project s/Ferroelectrics/IMS2000\%20Workshop/WFE002.pdf

15. D. Webb, "Applications of Ferroelectrics in Military Systems," IMS2000; see also http: //my.ece.ucsb.edu/yorklab/Projects/Ferroelectrics/IMS2000\%20Workshop/WFE004.pdf

16. A.B. Kozyrev et al. Technical Physics Letters, v. 24, pp.19-25, (1998).

17. A. Kanareykin, A. Dedyk. S.F. Karmanenko, E. Nenasheva, and V.P. Yakovlev, "LowLoss Ferroelectrics for Accelerator Application,” EPAC2006, Edinburgh, June 26-30, 2006, pp. 3251-3253.

18. A. Kanareykin, private communications. 


\section{Omega-P, Inc.}

Final report submitted to DoE on Phase II SBIR grant DE-FG02-06 ER 84472

FAST FERROELECTRIC L-BAND TUNER FOR ILC CAVITIES

19. O.A. Nezhevenko, V.P. Yakovlev, "Cumulative Interaction in the Deflection Cavities of a High-Power Magnicon Amplifier," IEEE Transactions On Plasma Science, vol. 28, No. 3, June, 2000, pp 542-549.

20. D. Wang, Y. Zhao, I. Ben-Zvi, X. Chang, J. Kewich, Ch. Montag, J. Zekutovicz, C. Pagani, P. Pierini, "Design of a Superconducting Linac Cavity for High-Current Energy Recovery Linac Operation," PAC2003, Portland, May 11-16, 2003, pp. 1300-1302.

21 M. Liepe and S. Belomestnykh,"RF Parameters and Field Stability Requirements for the Cornell ERL Prototype", PAC2003, Portland, May 11-16, 2003, pp.1326-1328.

22. M. Liepe and S. Belomestnykh, J. Dobbins, R. Kaplan, C. Strohman, B. Stuhl, C. Hovater, T. Plawski, "Pushing the Limits: Rf Field Control at High Loaded Q", PAC2005, Knoxville, May 16-20, 2005, p. 2642-2644.

23. S. Belomestnykh, R. Kaplan, J. Reilly, V. Veshcherevich, "Instability of the Control Loop in the Presence of a High-Q passive superconductive cavity," PAC2005, Knoxville, May 16-20, 2005, p. 2553-2555.

24. Y. Kang, J. Wilson, M. McCartney, S-H. Kim, M. Champion, R. Kustom, B. Chase, "Development of TEM High Power RF Vector Modulators", CWHAP06, May 1-4, 2006, Argonne, IL.

25. V. Katalev, S. Choroba, "Tuning of External Q and Phase for the Cavities of a Superconducting Linear Accelerator", LINAC-2004, Lübeck, Germany, 2004, pp.724726.

26. I. Ben-Zvi, A. Todd, "Fully Superconducting Gun", AES-BNL meeting, Upton, July 22, 2004, http://www.bnl.gov/cad/ecooling/docs/PDF/Cryomodule/AlanTodd-FullSRFGun. pdf

27. S.Yu. Kazakov, V.P. Yakovlev, J.L. Hirshfield, A.D. Kanareykin, E.A. Nenasheva, "Fast Ferroelectric Tuner For L-Band," $12^{\text {th }}$ Advanced Accelerator Concepts Workshop (AAC2006), Grand Geneva Resort, July 10-15, 2006, see http://www.hep.anl.gov/ aac06/.

28. V.P. Yakovlev, O.A. Nezhevenko, J.L. Hirshfield, "Fast X-Band Phase Shifter," AAC2004, Stony Brook, June 2004, AIP Conference Proc. 737, Melville, N.Y., 2004, pp.643-650.

30. A. Kanareykin, E. Nenasheva, V. Yakovlev, A. Dedyk, S. Karmanenko, A. Kozyrev, V. Osadchiy. "Advanced Ferroelectric Material Development for Accelerator Component Applications," $12^{\text {th }}$ Advanced Accelerator Concepts Workshop (AAC2006), Grand Geneva Resort, July 10-15, 2006, see http://www.hep.anl.gov/aac06/. 